\title{
Model selection and validation of extreme distribution by goodness-of-fit test based on conditional position
}

\begin{abstract}
In Extreme Value Theory, the important aspect of model extrapolation is to model the extreme behavior. This is because the choice of the extreme value distribution model affects the prediction that is about to be made. Thus, model validation which is called Goodness-offit (GoF) test is necessary. In this study, the GoF tests were used to fit the Generalized Extreme Value (GEV) Type-II model against the simulated observed values. The $\varepsilon$, $\stackrel{\circ}{\mathrm{a}}$ and $\mathfrak{r}^{\mathrm{r}}$ were estimated by Maximum Likelihood. The critical values based on conditional points were developed by Monte-Carlo simulation. The powers of the tests were identified by power study. The data that is distributed according to GEV Type-II distribution was used to test whether the critical values developed are able to confirm the fit between GEV Type-II model and the data. To confirm the fit, the statistics value of the GOF test should be smaller than the critical value.
\end{abstract}

Keyword: Goodness-of-fit; Critical values; Power; Conditional position 